\title{
Human Umbilical Cord Blood-Derived Mesenchymal Stem Cells Upregulate Myelin Basic Protein in Shiverer Mice
}

\author{
Krishna Kumar Veeravalli, ${ }^{1, *}$ Venkata Ramesh Dasari, ${ }^{1, *}$ Daniel Fassett, ${ }^{2}$ Dzung H. Dinh, ${ }^{2}$ and Jasti S. Rao ${ }^{1,2}$
}

Human umbilical cord blood is a rich source of pluripotent mesenchymal stem cells and possesses significant advantages over other stem cell sources such as the embryo and bone marrow. In the present study, we aimed to investigate the potential of human umbilical cord blood-derived pluripotent mesenchymal stem cells (hUCB) to myelinate the axons of shiverer mice brains. We also investigated the effect of hUCB treatment on regulation of myelin basic protein in vitro in PC-12 cells, which are normally not myelinated. The results of our study clearly demonstrated that hUCB survive and migrate in vivo and has the potential to myelinate shiverer mice brains. The expression level of myelin basic protein, a major component of the myelin sheath, has been significantly increased in vivo and in vitro as revealed by Western blot, reverse transcription (RT)-polymerase chain reaction, immunohistochemistry, immunocytochemistry, and fluorescent in situ hybridization results. Further, transmission electron microscopic images of hUCB-treated shiverer mice brains showed several layers of myelin around the axons compared with a thin and fragmented layer of myelin in untreated animals. Moreover, the frequency of shivering was diminished 1 month after hUCB treatment in shiverer mice. Our results strongly indicated that hUCB transplantation could be an effective means of treating demyelinating or hypomyelinating disorders.

\section{Introduction}

$\mathrm{C}$ URRENTLY, BONE MARROW REPRESENTS the main source of mesenchymal stem cells (MSCs) for both experimental and clinical studies [1-3]. Human umbilical cord blood is often used as an alternative source of stem or progenitor cells [4]. Unlike bone marrow-derived MSCs, stem cells from human umbilical cord blood like placental blood can be obtained without risk to mother or infant [5]. In spite of the small numbers of MSCs obtainable from cord blood in comparison to bone marrow [6], human umbilical cord blood progenitor cells were shown to possess significant advantages over bone marrow in terms of proliferative capacity and immunologic reactivity [7]. Further, cord blood stem cells are more immature than adult MSCs and expand readily in vitro [8]. Owing to their primitive nature and ability to develop into nonhematopoietic cells of various tissue lineages, including neural cells, human umbilical cord blood-derived pluripotent mesenchymal stem cells (hUCB) may be useful as an alternative cell source for cell-based therapies requiring the replacement of individual cell types and/or substitution of missing substances [9]. Although the MSCs derived from Wharton's jelly of the umbilical cord have similar advantages, their culture expansion has a dis- advantage, in that the cells cannot be frozen on the same day as hUCB arrive in the laboratory, and there is the increased risk of contamination with any culture manipulation [10]. Given these features along with their potent differentiation potential [11], hUCB are an attractive source for cellular or gene transfer therapy.

Previous studies have reported that hUCB are beneficial in reversing the behavioral effects after spinal cord injury (SCI), even when infused 5 days after injury [12]. Moreover, a female patient with chronic SCI showed functional and morphological improvement after hUCB treatment [13]. Transplanted hUCB differentiate into various neural cells and induce motor function improvement in cord-injured rat models [14]. However, it is unclear whether the enhanced functional recovery results from remyelination of demyelinated axons by engrafted cells or by trophic support to spare the white matter that would otherwise degenerate. Recent studies from our laboratory have demonstrated that hUCB, when transplanted into the spinal cord 7 days after weight-drop injury, survived for at least 2 weeks, differentiated to oligodendrocytes, and enhanced myelinogenesis $[15,16]$.

Congenitally hypomyelinated shiverer mice fail to generate compact myelin and die by 18-21 weeks of age. Myelin sheaths in these mice are slightly thinner than normal in

Departments of ${ }^{1}$ Cancer Biology and Pharmacology and ${ }^{2}$ Neurosurgery, University of Illinois College of Medicine at Peoria, Peoria, Illinois.

*These two authors contributed equally to this work. 
peripheral nervous system (PNS) $[17,18]$ compared with markedly deficient myelin in central nervous system (CNS) $[19,20]$. Hence, in the present study, we aimed to investigate the capability of hUCB to myelinate the axons of shiverer mice brains. We also investigated the effect of hUCB on myelin basic protein (MBP) regulation in vitro in PC-12 cell cultures. Our results clearly demonstrated that hUCB have the potential to myelinate axons of shiverer brains and the findings of this study strongly indicated that hUCB transplantation could be an effective means of treating demyelinating or hypomyelinating disorders.

\section{Materials and Methods}

\section{Culture of hUCB and WI-38 cells}

Human umbilical cord blood was collected from healthy volunteers with informed consent and according to a protocol approved by the Institutional Review Board. Cord blood was enriched by sequential Ficoll density gradient purification. Next, we selected cells using CD29 ${ }^{+}, \mathrm{CD} 44^{+}$, $\mathrm{CD} 81^{+}$, and $\mathrm{CD}_{133^{+}}$markers. The nucleated cells were grown in Mesencult basal medium (Stem Cell Technologies) supplemented with $20 \%$ heat inactivated FBS (Hyclone) and $1 \%$ penicillin and streptomycin (Invitrogen). Stem cells were incubated at $37^{\circ} \mathrm{C}$ in an incubator with $5 \% \mathrm{CO}_{2}$ at saturating humidity. Fluorescence-activated cell sorting (FACS) and immunofluorescence analysis revealed that $\mathrm{LUCB}$ used in the present study were positive for CD29, CD81, CD133 and CD44 (Supplementary Fig. S1; Supplementary Data are available online at www.liebertonline.com/scd). WI-38 cells (normal human lung fibroblasts) obtained from ATCC were cultured at $37^{\circ} \mathrm{C}$ and $5 \% \mathrm{CO}_{2}$ in Eagle's minimum essential medium containing $10 \%$ FBS. During the experiments, the medium from culture plates was changed every 2 days.

\section{Intracranial implantation of hUCB and WI-38 cells}

Female shiverer mice were used for the study. The Institutional Animal Care and Use Committee of the University of Illinois College of Medicine at Peoria approved all surgical interventions and postoperative animal care. Study design, group description, and the number of animals used for various experiments in the study are depicted in Table 1. Mice (6-9 weeks old) were anesthetized with intraperitoneal injections of ketamine $(50 \mathrm{mg} / \mathrm{kg})$ and xylazine $(5 \mathrm{mg} / \mathrm{kg})$. A 2 to $3 \mathrm{~mm}$-long incision was made in the cerebrum just to the right of midline and anterior to the interaural line so that the coronal and sagittal sutures could be identified and the guide screw entry site was marked at a point $2.5 \mathrm{~mm}$ lateral and $1 \mathrm{~mm}$ anterior to the bregma. Using a small hand-controlled drill, a hole was made in the animal's skull. Ten microliters of cell suspension containing $2 \times 10^{5}$ hUCB or WI-38 cells in PBS was injected $(1 \mu \mathrm{L} / \mathrm{min})$ intracerebrally using a Hamilton syringe as per the study design. After the entire volume of the cell suspension was injected, the needle was removed slowly and bone wax was applied to close the system. Control animals were injected intracerebrally with $10 \mu \mathrm{L}$ PBS.

To observe intracranial migration, a few animals were implanted with hUCB and WI-38 cells labeled, respectively, with Qtracker ${ }^{\circledR} 655$ (red fluorescence) and Qtracker 525 (green fluorescence) cell-labeling kits (Invitrogen). Cyclosporine A (10 mg/kg; Bedford Labs) was administered till 1

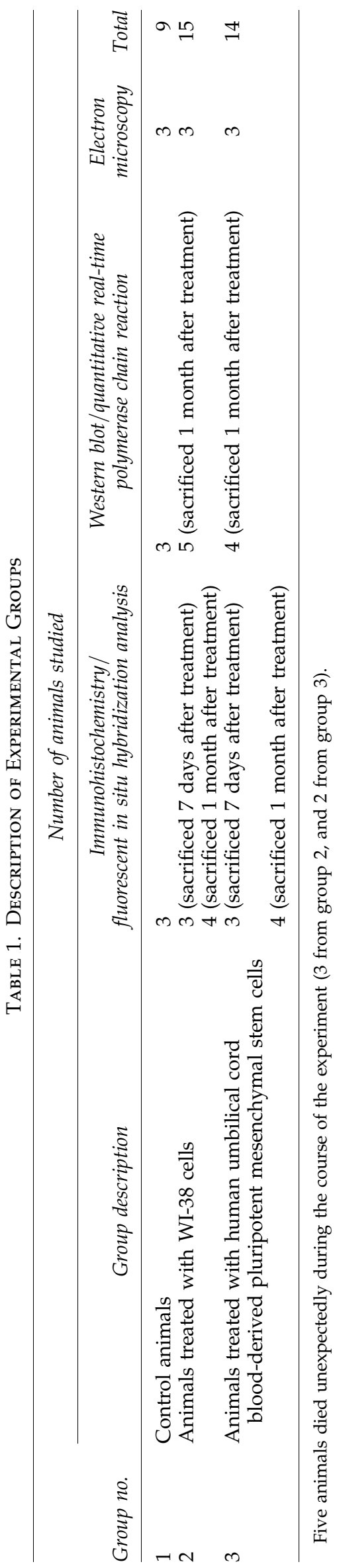


week to avoid rejection after hUCB or WI-38 transplantation. Mice were sacrificed on day 7, brains harvested, brains freehand sectioned (coronal sections) at regions adjacent to and covering the site of implantation, and sections were observed under a fluorescent dissection microscope (Olympus SZX 12) to observe hUCB tracker red and WI-38 tracker green fluorescence.

\section{Western blot analysis}

Mice were euthanized, and brain tissues covering the site of injection were isolated and suspended in $0.5 \mathrm{~mL}$ of cell lysis buffer [ $50 \mathrm{mM}$ Tris- $\mathrm{HCl}, 150 \mathrm{mM} \mathrm{NaCl}, 1 \%$ of Igepal, $1 \mathrm{mM}$ ethylenediaminetetraacetic acid, $1 \mathrm{mM} \mathrm{NaF}, 1 \mathrm{mM}$ $\mathrm{Na}_{3} \mathrm{VO}_{4}$, and $1 \mathrm{mM}$ PMSF plus protease inhibitors $(1 \mu \mathrm{g} / \mathrm{mL}$ pepstatin, $1 \mu \mathrm{g} / \mathrm{mL}$ leupeptin, and $1 \mu \mathrm{g} / \mathrm{mL}$ aprotinin), $\mathrm{pH}$ of 7.4] and homogenized. Samples $(50 \mu \mathrm{g}$ of total protein/well) were subjected to $12 \%$ SDS-PAGE, and the protein bands on the gel were transferred onto nitrocellulose membranes. The membranes were processed with mouse monoclonal MBP primary antibody (BD Biosciences Pharmingen) followed by a horseradish peroxidase (HRP)conjugated secondary antibody. Glyceraldehyde 3-phophate dehydrogenase antibody was used to verify that similar amounts of protein were loaded in all lanes.

\section{RNA extraction and reverse transcription- polymerase chain reaction}

Brains of animals from various groups were homogenized and total RNAs were isolated. Approximately $1 \mu \mathrm{g}$ of total RNA from each sample was reverse transcribed into cDNA as per the manufacturer's instructions using the iScript ${ }^{\mathrm{TM}}$ cDNA Synthesis Kit (Bio-Rad Laboratories, CA). We used the following sequences for the forward and reverse primers:

For MBP:

Forward primer - 5'CTCTGGCAAGGACTCACACA3'

Reverse primer - 5'GTCTCTTCCTCCCCAGCTA3'

For $\beta$-actin:

Forward primer - 5'GTCGTACCACTGGCATTGT3'

Reverse primer - 5'CAGCTGTGGTGGTGAAGCT3'

Samples were subjected to 40 cycles at $95^{\circ} \mathrm{C}$ for $30 \mathrm{sec}$, $60^{\circ} \mathrm{C}$ for $30 \mathrm{sec}$, and $72^{\circ} \mathrm{C}$ for $1 \mathrm{~min}$ on GeneAmp PCR System 9700 (Perkin Elmer, Boston, MA) in $25 \mu \mathrm{L}$ reaction volumes. After amplification, reverse transcription (RT)-polymerase chain reaction (PCR) products were separated on a $1 \%$ agarose gel containing $0.5 \mathrm{mg} / \mathrm{mL}$ ethidium bromide.

\section{Quantitative real-time PCR}

cDNAs synthesized from total RNAs of the brain tissues of control, WI-38-treated, and hUCB-treated mice were also used for real-time PCR analysis. Reaction setup for each sample (a final volume of $25 \mu \mathrm{L}$ ) was put together using the IQ $^{\text {TM }}$ SYBR ${ }^{\circledR}$ Green Supermix kit (Bio-Rad Laboratories, CA) as per the manufacturer's instructions. Samples were subjected to 40 cycles at $95^{\circ} \mathrm{C}$ for $30 \mathrm{sec}, 60^{\circ} \mathrm{C}$ for $30 \mathrm{sec}$, and $72^{\circ} \mathrm{C}$ for $1 \mathrm{~min}$ in iCycler IQ (Multi Color Real-Time PCR Detection System; Bio-Rad Laboratories). Experiments were conducted with brain samples of 3 different animals from each group and in triplicates for MBP and $\beta$-actin. Data were collected and recorded using iCycler IQ software (Bio-Rad Laboratories) and expressed as a function of the threshold cycle $(\mathrm{Ct})$, which represents the number of cycles at which the fluorescent intensity of the SYBR Green dye is significantly above the background fluorescence. Average $\mathrm{Ct}$ values from triplicate samples were normalized from average $\mathrm{Ct}$ values of $\beta$-actin.

\section{Immunohistochemical assessment}

Mice were deeply anesthetized with ketamine/xylazine as described earlier and intracardially perfused with $4 \%$ paraformaldehyde in $0.1 \mathrm{M}$ phosphate buffer ( $\mathrm{pH}$ 7.4). Brains of the animals from various treatment groups were removed, fixed in $10 \%$ buffered formalin, and embedded in paraffin. Serial cross sections ( $5 \mu \mathrm{m}$ thick) of the brains were obtained with a microtome. The sections were processed with mouse monoclonal MBP primary antibody (Santa Cruz Biotechnology; 1:100 dilution), HRP-conjugated secondary antibody, and $3,3^{\prime}$ diamino benzidine (DAB; Sigma-Aldrich). Slides were stained with hematoxylin to observe the nucleus, mounted, and observed under a light microscope. Fields with brown stain resulting from DAB interaction were observed for MBP expression.

To address the in vivo survival of hUCB, immunohistochemical analysis was performed on the brain sections of control, WI-38-implanted, and hUCB-implanted shiverer mice. Four weeks after implantation, mice were deeply anesthetized and intracardially perfused with $4 \%$ paraformaldehyde in $0.1 \mathrm{M}$ phosphate buffer ( $\mathrm{pH} 7.4$ ), and brains were removed, fixed in $10 \%$ buffered formalin, and embedded in paraffin. Paraffin sections were processed for immunohistochemical analysis with CD29 (Santa Cruz Biotechnology), CD44 (Cell Signaling), and CD81 (Santa Cruz Biotechnology) antibodies followed by Alexa Fluor fluorescent-labeled secondary antibodies.

\section{Fluorescent in situ hybridization analysis}

For mRNA in situ hybridization, we followed the method of Wrathall et al. [21] with slight modifications. Paraffinembedded cross sections ( $5 \mu \mathrm{m}$ thick) of the brains obtained from control, WI-38-treated, and hUCB-treated groups of animals were used for in situ hybridization. The following oligonucleotide antisense sequences with a length of 48 bases were used as probes for MBP gene:

MBP sense: ATGGCATCACAGAAGAGACCCTCACAG CGACACGGATCCAAGTACTTG

MBP antisense: CAAGTACTTGGATCCGTGTCGCTGT GAGGGTCTCTTCTGTGATGCCAT

The oligonucleotides were labeled with FITC at $3^{\prime}$ ends (Sigma-Genosys, The Woodlands, TX). For in situ hybridizations, slides were deparaffinized, permeabilized, and acetylated $(0.25 \%$ acetic anhydride in $0.1 \mathrm{M}$ triethanolamine $\mathrm{HCl}, \mathrm{pH} 8$, for $10 \mathrm{~min})$. They were then incubated for $2 \mathrm{~h}$ at $37^{\circ} \mathrm{C}$ in prehybridization buffer $[50 \%$ formamide, $5 \times$ saline sodium citrate buffer (SSC), $5 \times$ Denhardt's solution (1\% BSA, $1 \%$ Ficoll, and $1 \%$ Polyvinyl pyrrolidone), $0.025 \%$ bakers yeast tRNA (Sigma), and $0.05 \%$ herring sperm DNA (Sigma)] followed by overnight incubation at $37^{\circ} \mathrm{C}$ with hybridization buffer (prehybridization buffer containing $200 \mathrm{ng} / \mathrm{mL}$ of each oligonucleotide probe). The next day, slides were washed sequentially with $2 \times \operatorname{SSC}(0.15 \mathrm{M} \mathrm{NaCl}$, and $15 \mathrm{mM}$ sodium citrate, $\mathrm{pH} 7.0$ ) for $5 \mathrm{~min}$ at room temperature, $0.2 \times \mathrm{SSC}\left(1 \mathrm{~h}\right.$ at $72^{\circ} \mathrm{C}$ in shaking water bath $)$ and $0.2 \times \operatorname{SSC}(5 \mathrm{~min}$ at room temperature) and then allowed for 
detection of fluorescent-labeled probes using ELF-97 mRNA in situ hybridization kit (Invitrogen). Finally, the slides were counterstained with Hoechst 33342 (for observation of cell nuclei) and mounted using the mounting medium. Observation of fluorescent in situ hybridization (FISH) signal was carried out with a confocal microscope (Olympus Fluoview). Sections stained with sense probes served as controls, which did not show any signal.

\section{Transmission electron microscopic studies}

To further characterize the myelination around the axons of the control, WI-38-implanted, and hUCB-implanted mice brains, at the end of the study, animals were anesthetized and perfused with $4 \%$ paraformaldehyde followed by a fixative solution ( $2 \%$ glutaraldehyde, $2 \%$ paraformaldehyde, and $2 \mathrm{mM} \mathrm{CaCl}_{2}$ in $0.1 \mathrm{M}$ cacodylate buffer, $\mathrm{pH}$ 7.3). Onemicrometer sections were cut from the various portions of the brain with glass knives on an ultramicrotome, stained with toluidine blue, and examined under light microscopy. After fixation with $2.5 \%$ glutaraldehyde, the samples were postfixed with $1 \%$ osmium tetroxide, dehydrated, and flat embedded in Epon 812 epoxy resin (Tousimis). A Reichert OMU3 ultramicrotome (Austria) was used to prepare $600 \AA$ thin sections that were mounted on 200 mesh copper grids, stained with uranyl acetate and lead citrate. The sections were viewed under a JEOL JEM 100C transmission electron microscope.

\section{Cultivation of PC-12 cells}

PC-12 cells (rat pheochromocytoma cell line) obtained from ATCC were grown in F12K medium supplemented with $2.5 \%$ FBS and $15 \%$ horse serum in a humidified atmosphere containing $5 \% \mathrm{CO}_{2}$ at $37^{\circ} \mathrm{C}$. During the experiments, the medium from culture plates was changed every 2 days.

\section{In vitro differentiation of hUCB}

When hUCB reached $70 \%-80 \%$ confluency, cells were detached with trypsin-ethylenediaminetetraacetic acid and centrifuged at $250 \mathrm{~g}$ for $3 \mathrm{~min}$ and replated. An acclimatization step was carried out 2 days before oligodendrocyte induction by replacing the growth medium with preinduction medium consisting of Neurobasal medium (Invitrogen) supplemented with 10\% FBS (Hyclone), 1\% penicillinstreptomycin (Invitrogen), 1\% $200 \mathrm{mM}$ L-glutamine (Mediatech, Inc.-Fisher), 2\% B27 (Invitrogen), 1\% N2 (Invitrogen), hEGF (10 ng/mL; Sigma), bFGF (10 ng/mL; Invitrogen), $\beta$-NGF (10 ng/mL; Sigma), BDNF $(10 \mathrm{ng} / \mathrm{mL} ;$ EMD Biosciences), and NT-3 (10 ng/mL; EMD Biosciences). After 2 days, differentiation of hUCB to oligodendrocytes was initiated by incubating the cells in induction medium consisting of preinduction medium without growth factors and with tri-idothyronine $(30 \mathrm{ng} / \mathrm{mL})$. The cells were observed for differentiation for 10 days.

\section{Cocultures}

PC-12 cells in a $40 \%$ confluent plate were lifted by incubation with TrypLE Express (Invitrogen) at $37^{\circ} \mathrm{C}$ for $5 \mathrm{~min}$ and added to $50 \%$ confluent differentiated (to oligoden- drocytes) hUCB plates. Similarly, cells from $40 \%$ confluent PC-12 cell plate were added to 50\% confluent WI-38 plate. The cocultures of hUCB or WI-38 cells with PC- 12 cells were grown in a humidified atmosphere containing $5 \% \mathrm{CO}_{2}$ at $37^{\circ} \mathrm{C}$ supplemented with $\mathrm{F} 12 \mathrm{~K}$ medium containing $2.5 \%$ FBS and $15 \%$ horse serum. The cocultures were maintained for 5 days and 1 half of the medium was changed every 2 days.

\section{Immunocytochemistry}

PC-12 cells, cocultures of PC-12 with WI-38 cells, and PC12 cells with transdifferentiated (to oligodendrocytes) hUCB cultured in 2-well slides for 5 days were washed with PBS and fixed with $10 \%$ buffered formalin phosphate. The cells were permeabilized with $0.3 \%$ TritonX-100 in PBS, and blocked with $1 \%$ BSA in 1XPBS followed by incubations in mouse monoclonal MBP primary antibody (Santa Cruz Biotechnology) and goat anti-mouse HRP-conjugated secondary antibody. The sections were washed in PBS and incubated with DAB for MBP staining and then the nuclei were counterstained with Hoechst 33342.

\section{Densitometry}

Densitometry was performed using ImageJ software (National Institutes of Health) to quantify the band intensities obtained from Western blot analysis and agarose gel electrophoresis. Data represent average values from 3 independent experiments.

\section{Statistical analysis}

Statistical comparisons were performed using Graph Pad Prism software (version 3.02). Quantitative data from realtime PCR, Western blot analysis, and agarose gel electrophoresis were evaluated for statistical significance using 1-way ANOVA. Bonferroni's post hoc test (multiple comparison tests) was used to compare statistical significance between groups. Differences in the values were considered significant at $P<0.05$.

\section{Results \\ $M B P$ expression increases in vivo after hUCB treatment}

Western blot analysis was performed with the samples collected from the brain tissues of control, WI-38-treated, and hUCB-treated shiverer mice (1 month) to evaluate expression of MBP. More prominent and dense band representing expression of MBP at $18.5 \mathrm{kDa}$ was noticed in hUCB-treated samples compared with barely visible bands in control and WI-38-treated samples (Fig. 1A). One more prominent band representing $\mathrm{MBP}$ was also noticed in hUCB-treated samples at $21.5 \mathrm{kDa}$. Similar band patterns were noticed for the MBP gene when the cDNAs of control, WI-38-treated, and hUCB-treated samples were reverse transcribed followed by PCR and agarose gel electrophoresis (Fig. 1B). Densitometric analysis of the bands obtained in both Western blot and reverse transcription PCR indicated significant increase in expression of MBP compared with control and WI-38-treated samples (Fig. 1C, D). Further, real-time PCR analysis of the reverse-transcribed 


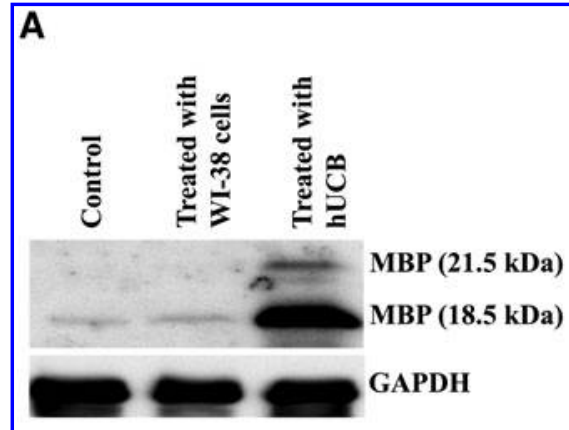

B

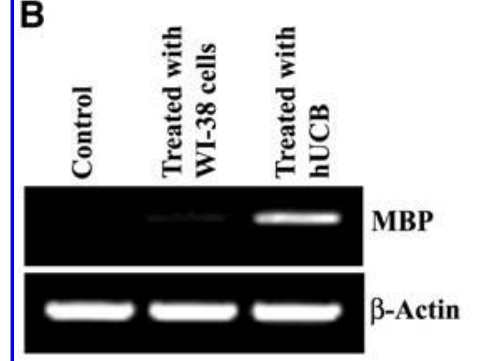

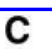

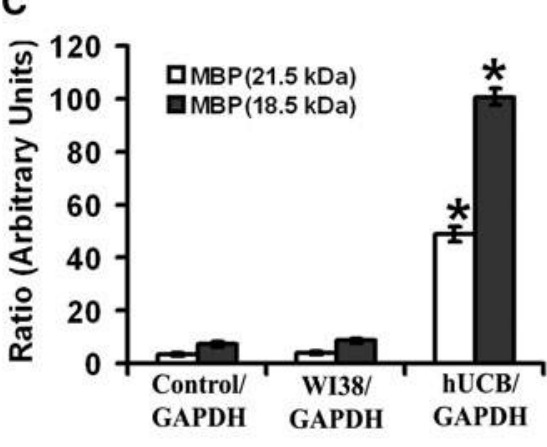

D

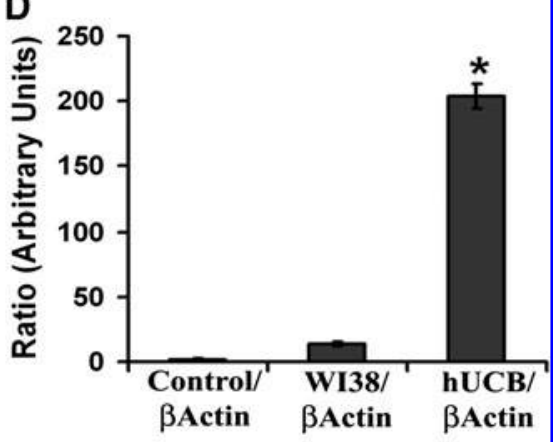

FIG. 1. Western blot analysis (A) and RT-polymerase chain reaction (PCR) analysis (B) showing the changes in myelin basic protein (MBP) levels in control, WI-38-implanted, and human umbilical cord bloodderived pluripotent mesenchymal stem cell ( $h U C B)$-implanted shiverer mice brains. Glyceraldehyde 3phophate dehydrogenase (GAPDH) and $\beta$-actin were used as loading controls for Western blot and RT-PCR analysis, respectively. These images are representative of 3 different experiments performed with 3 different shiverer mice brain samples $(n \geq 3)$. C and $\mathbf{D}$ represent the corresponding quantitative analysis of bands for MBP using ImageJ software. Error bars indicate SEM. ${ }^{*} P<0.05$ compared with control values. (E) Quantitative real-time PCR data of MBP in the amplified cDNAs obtained from the shiverer mice brains of control, WI-38-implanted, and hUCB-implanted animals. MBP upregulation was significant in hUCB-implanted shiverer mice. Error bars indicate SD. $n=3$ (number of samples from each group). ${ }^{*} P<0.05$ versus control values.

cDNAs obtained from control, WI-38-treated, and hUCBtreated samples revealed statistically significant upregulation of MBP in hUCB-treated samples as compared with controls (Fig. 1E). The upregulation of MBP mRNA in hUCB-treated samples was almost 44-fold more than the controls, whereas MBP levels in WI-38-treated samples were comparable to that of controls. To further confirm these results, we performed immunohistochemical analysis of brain sections collected from various groups. The brain sections of the animals obtained after treatment with hUCB showed prominent dark brown staining representing high expression of MBP (Fig. 2A). We do not see MBP expression either in control or in WI-38-treated brain sections of shiverer mice (Fig. 2A). These data were in agreement with the results of Western blot and RT-PCR analyses.

\section{FISH analysis and electron microscopic studies}

To demonstrate the myelination of shiverer mice brains after hUCB treatment, we determined mRNA levels of MBP using the FISH technique. Paraffin sections of shiverer mice brains from control, WI-38-treated, and hUCB-treated groups were hybridized with FITC-conjugated MBP sense and MBP antisense oligonucleotides. Green fluorescence indicative of MBP mRNA expression was noticed in hUCBtreated sections hybridized with $\mathrm{MBP}$ antisense oligonucleotide (Fig. 2B). No green fluorescence was noticed in control and WI-38-treated sections hybridized with MBP antisense oligonucleotide (Fig. 2B). Similarly, no green fluorescence was noticed in hUCB-treated sections hybridized with MBP sense oligonucleotide (Fig. 2C). The results of FISH analysis were in agreement with the results of the Western blot, RT-PCR, and immunohistochemical analyses, which further confirmed the upregulation of MBP by hUCB in vivo in shiverer mice. Further, as revealed by transmission electron microscopic images, myelination around the axons of the hUCB-treated shiverer mice brains is prominent compared with very thin myelinated axons in control and WI-38-implanted mice (Fig. 3). Magnified transmission electron microscopic images clearly demonstrate the presence of several layers of myelin around the axons after hUCB treatment (Fig. 3).

\section{In vivo survival and migration of hUCB}

To determine the migration capability of hUCB under in vivo conditions, we injected hUCB intracranially to shiverer mice as explained in Materials and Methods. One 
FIG. 2. (A) Immunohistochemistry of MBP in control, WI-38-implanted, and hUCB-implanted shiverer mice brains. Immunohistochemical comparison was performed to demonstrate the increases in MBP expression in hUCB-implanted shiverer brains (brown staining indicated by arrows). Paraffin sections of shiverer mice brains from various groups were probed with MBP antibodies using 3,3' diamino benzidine (DAB) immunohistochemistry, counterstained with hematoxylin to stain the nuclei, and then photographed using brightfield microscope. Results are from 3 independent sections $(n=3)$. (B, C) Pattern of mRNA expression of MBP in shiverer mice brains. Paraffin sections of shiverer mice brains from control, WI-38-implanted, and hUCB-implanted groups were processed for fluorescent in situ hybridization analysis to study MBP mRNA expression. Serial cross sections hybridized with FITCconjugated antisense oligonucleotide for MBP were photographed using confocal microscope. Green fluorescence indicative of MBP mRNA expression was observed in brain sections of hUCB-implanted animals hybridized with MBP antisense oligonucleotide (B). No green fluorescence was noticed with the use of MBP sense oligonucleotide in brain sections of hUCB-implanted animals (C). Cells were counterstained for nuclei with Hoechst33342. Images are representatives of the sections obtained from 3 different animals $(n=3)$. Color images available online at www.liebertonline.com/scd.

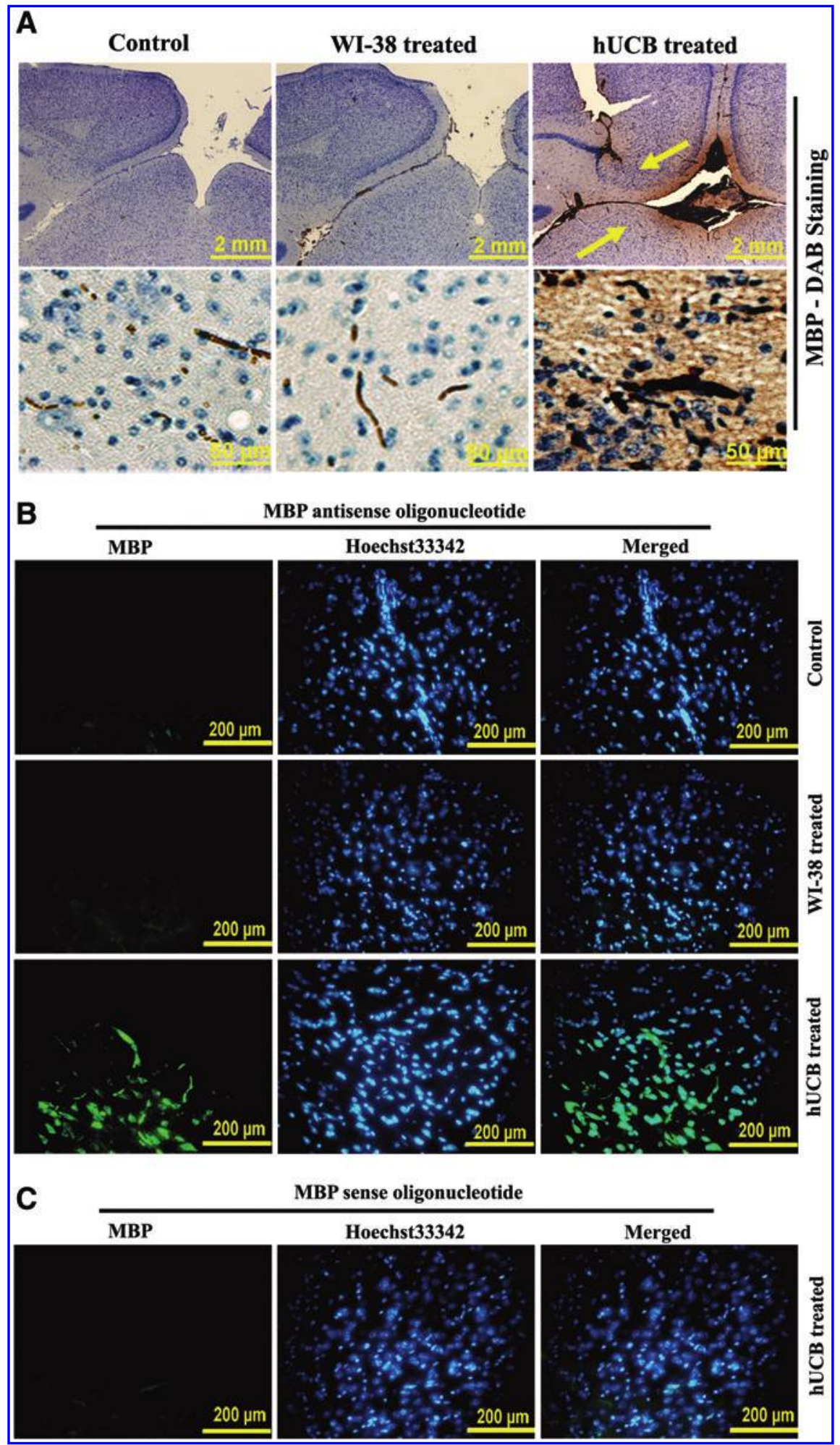

week after intracranial implantation of hUCB (labeled with Qtracker 655- red fluorescence) and WI-38 cells (labeled with Qtracker 525-green fluorescence) in shiverer mice, the mice were sacrificed and free hand sections of the brains at regions adjacent to and covering the site of implantation were observed under a fluorescent microscope to study the migrating potential of hUCB. WI-38 cells served as control cells to hUCB. We noticed slight green fluorescence only at the site of injection of the cells, whereas the red fluorescence indicative of hUCB was observed in several areas of the brain section (Fig. 4A), which indicates the survival and migration potential of hUCB in vivo in the brains of shiverer mice. Immunofluorescence analysis of brain sections 4 weeks after intracranial implantation also revealed a 


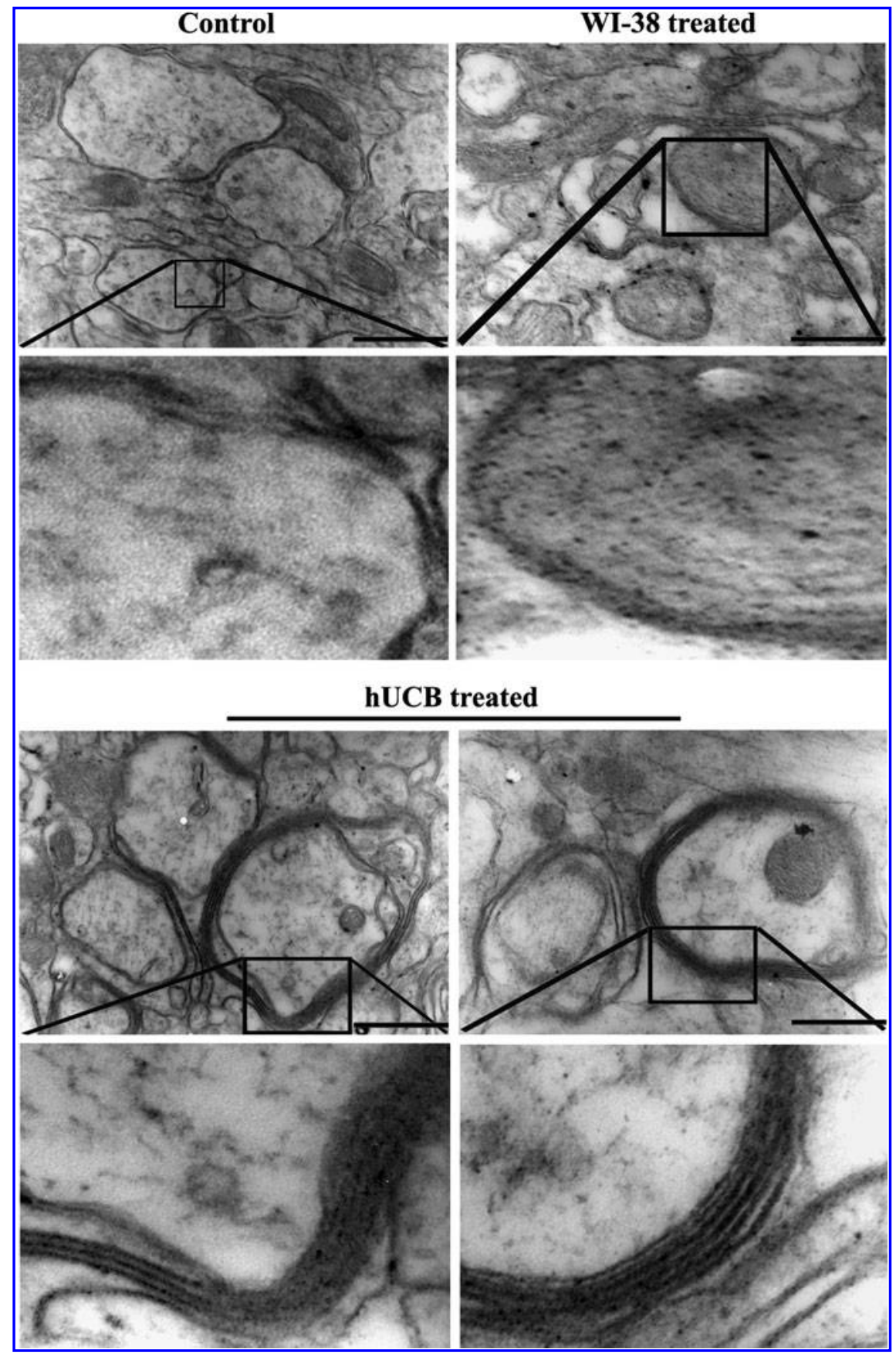

FIG. 3. Transmission electron micrographs of shiverer mice brains showing thin and fragmented myelin around the axons in control and WI-38-implanted mice. In contrast, hUCB-treated shiverer brains showing myelin with several layers. Images are representatives of the several sections obtained from 3 different animals $(n=3)$. Scale bar $=33,000 \times$.

robust survival of transplanted hUCB in shiverer mice brains (Fig. 4B).

\section{Upregulation of $M B P$ in vitro in PC12-cells}

To evaluate the potential of hUCB to synthesize or increase expression of MBP, we cocultured PC-12 cells with transdifferentiated hUCB (to oligodendrocytes) for 5 days (Fig. 5A) followed by immunocytochemical analysis with MBP antibody (Fig. 5B). To serve as a control to hUCB, we cocultured PC-12 cells with WI-38 cells and performed immunocytochemical analysis. As expected, we did not notice any brown staining in PC-12 cells and in cocultures of PC-12 with WI-38 cells (Fig. 5B). Interestingly, we observed prominent dark brown staining representative of MBP expression in the cocultures of PC-12 cells with transdifferentiated hUCB. Further, the cDNAs synthesized from the mRNAs of respective control, PC-12 with WI-38 coculture, and PC-12 with transdifferentiated hUCB cocultures were reverse transcribed and subjected to realtime PCR analysis to quantitate the folds upregulation of MBP. As expected and in concurrence with immunocytochemical analysis results, real-time PCR analysis revealed an 15-fold upregulation of MBP in the samples of PC-12 and hUCB cocultures compared with controls (Fig. 5C). These in vitro results are in accordance with our in vivo 
FIG. 4. (A) Bright-field images (A1, A4) depict the morphology of WI-38 cells and hUCB, respectively. A2 and A5 are the fluorescent images corresponding to Qtracker ${ }^{\circledR} 525$ (green fluorescence)-labeled WI-38 cells and Qtracker 655 (red fluorescence)labeled hUCB, respectively, which were intracranially implanted in shiverer mice brains. A3 and A6 represent the fluorescent images of shiverer mice brains that were free hand sectioned (at regions adjacent to and covering the site of implantation) on day 7 after implantation of WI-38 and hUCB, respectively. Compared with green fluorescence, which is indicative of WI-38 cells (A3, arrow shows fluorescence), the intensity of the red fluorescence is more (A6, arrows show red-fluorescence) and found at various regions in the brain, thereby indicating that intracranially implanted hUCB survived well and migrated from the site of injection to various regions of the brain in shiverer mice. (B) Survival of hUCB in shiverer mice brains (4 weeks after implantation). Brain sections of control, WI-38-treated, and hUCB-treated animals were processed for immunofluorescence with CD29 (red), CD44 (red), and CD81 (green) antibodies. Nuclei were stained with DAPI. $n=3$. Scale bar $50 \mu \mathrm{m}$. Color images available online at www.liebertonline.com/scd.

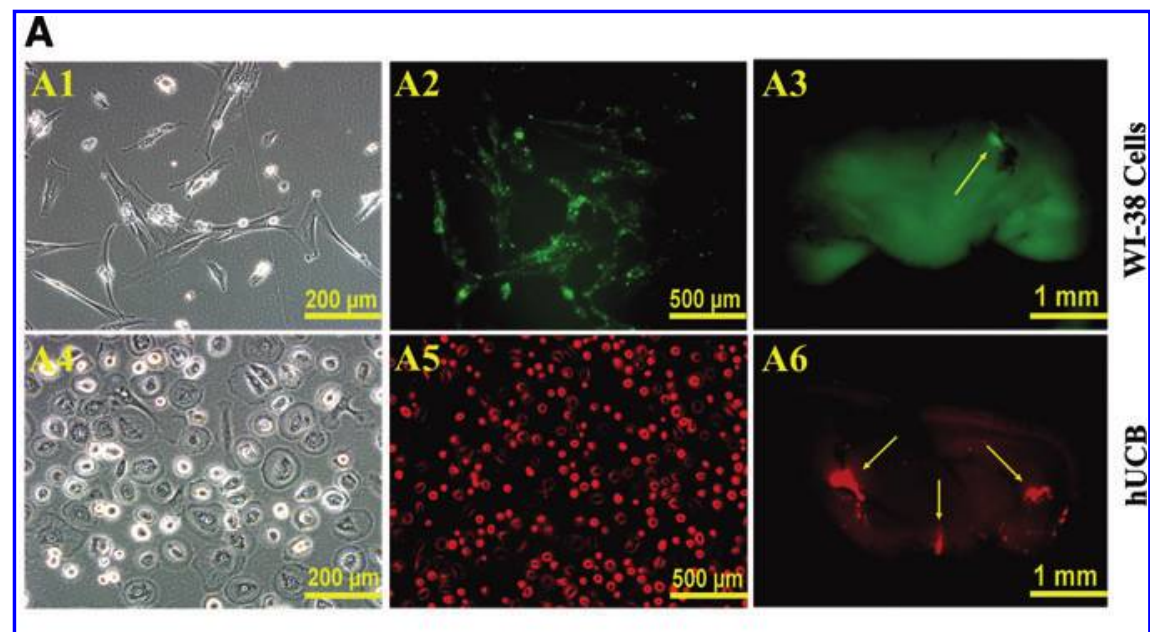

B
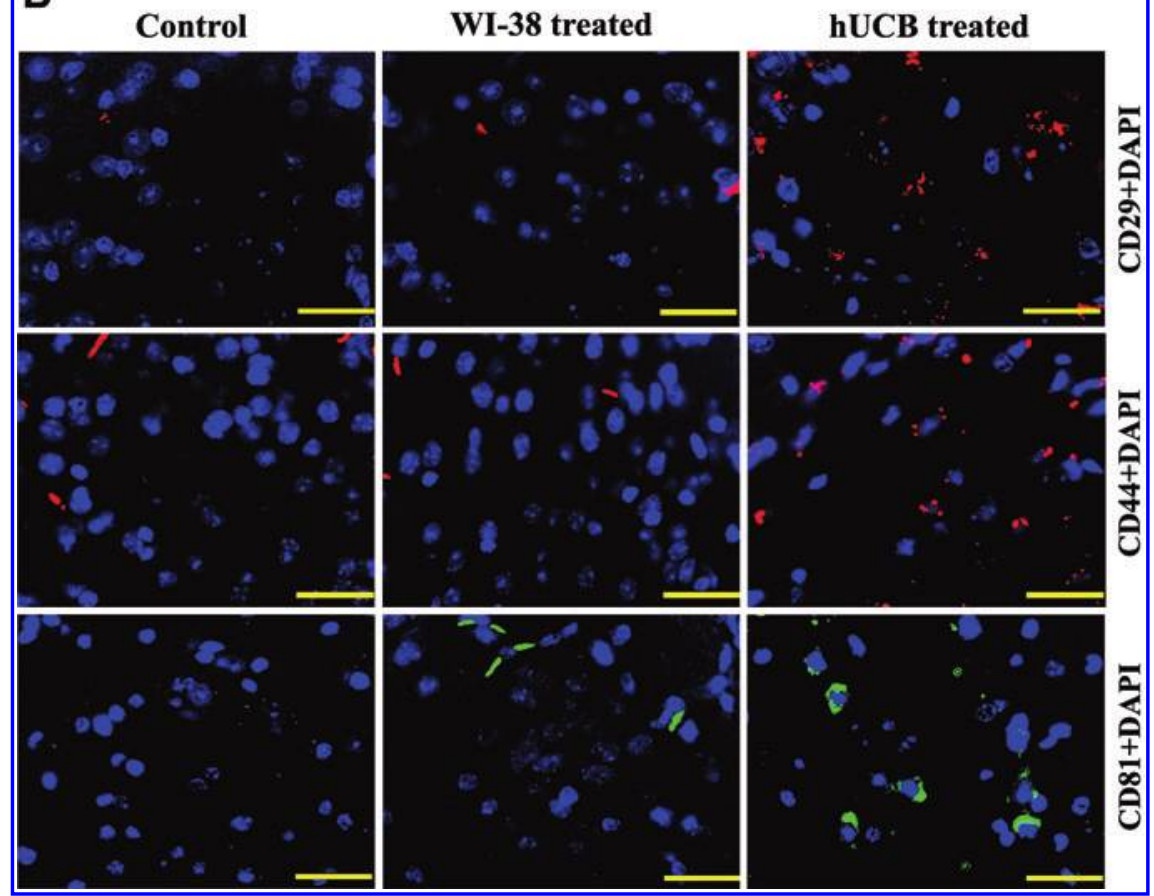

data and strongly suggest the potential of hUCB to upregulate MBP when implanted intracranially in shiverer mice.

\section{Discussion}

Even though neural stem cells and embryonic stem cell-derived glia were reported to give rise to myelinogenic oligodendrocytes in shiverer mice, they did not yield sufficient myelination to provide significant survival or long-term functional benefit [22-24]. In contrast, human glial progenitor cells implanted within a day of birth over 5 injection sites generated substantial myelin in shiverer mice and increased the life span and myelinated the central white matter of congenitally hypomyelinated mice $[25,26]$. However, only $23 \%$ of the transplanted shiverers were rescued by neonatal transplantation. Further, the improved ambulation with more forward motion and less retropulsion or freezing and diminished frequencies of seizures in these rescued mice were noticed at 7-8 months of their age. In the present study, intracerebral administration of hUCB as a single injection to shiverer mice of 6-9 weeks age resulted in a noticeable improvement in diminishing the frequency of seizures 1 month after their transplantation compared with controls (videos not shown). As expected, RT-PCR, real-time PCR, FISH analyses, Western blot, and immunohistochemical analysis revealed the post-transcriptional and post-translational elevations of MBP in hUCB-transplanted shiverer mice. These results are in agreement with our earlier findings wherein we have shown that the intraspinally implanted hUCB enhanced myelinogenesis and remyelinated the damaged axons in a rat model of spinal cord injury [15,27]. Transmission electron micrographs clearly have shown the axons surrounded by several layers of myelin in shiverer brains after hUCB transplantation compared with 


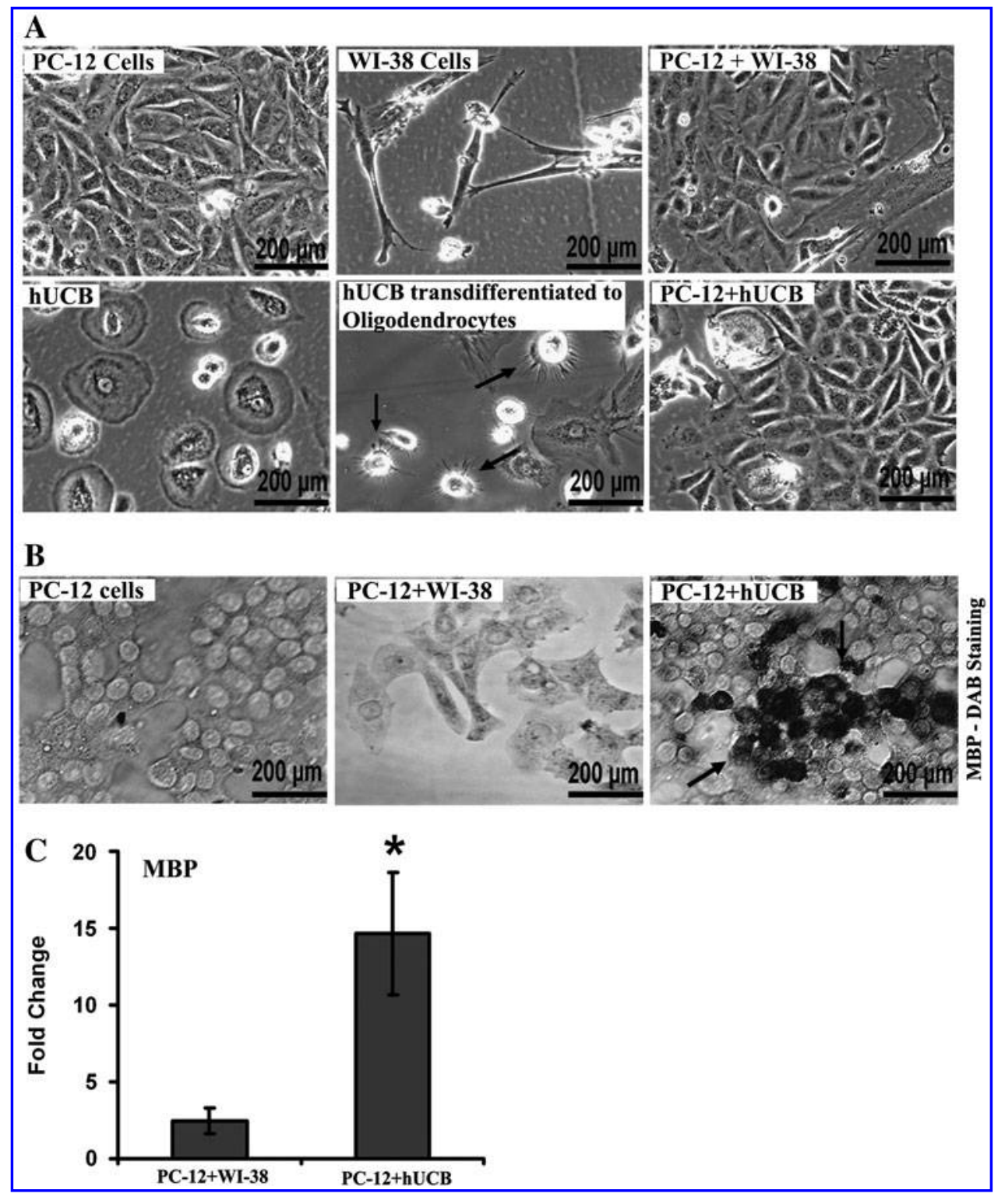

FIG. 5. (A) Bright-field images of PC- 12 cells, WI- 38 cells, hUCB, transdifferentiated hUCB, and the cocultures of PC-12 cells with WI-38 and transdifferentiated hUCB. Transdifferentiated hUCB showed morphology characteristic of oligodendrocytes, with flat cell body and short or long branched projections. Arrows show transdifferentiated hUCB. (B) PC-12 cells and cocultures of PC-12 cells with WI-38 and transdifferentiated hUCB were processed for MBP immunostaining using $3,3^{\prime}$ diamino benzidine and counterstained for nuclei with Hoechst 33342. Arrows show brown staining, which is indicative of MBP expression, that was observed in the cocultures of PC-12 cells with hUCB. Results are from 3 independent experiments $(n=$ 3). (C) Quantitative real-time PCR data of MBP in the amplified cDNAs obtained from PC-12 cells and their cocultures with WI-38 and hUCB. MBP upregulation was significant in PC-12 cells cocultured with hUCB. Error bars indicate $\mathrm{SD} . n=3 .{ }^{*} P<0.05$ versus control values.

untreated controls and further confirmed the myelinogenic potential of hUCB. The myelinogenic potential of hUCB could be attributed mainly to their transdifferentiating capability to various cell types. Recently, we have demonstrated the transdifferentiation of hUCB into adipo, osteo, and neural cells in vitro [28] and oligodendrocytes, astrocytes, and neurons in vitro and in vivo in a rat model [15]. As we know that it is possible to induce myelinating capacity of MSCs by chemically induced transdifferentiation [29], we treated hUCB that were transdifferentiated to oligodendrocytes, the myelinating cells of the CNS. Further, coculturing these transdifferentiated hUCB with PC12 cells significantly elevated both mRNA and protein levels of MBP in PC12 cells, which clearly indicated that the transdifferentiated oligodendrocytes are functional.

In addition to the myelinated axons and increased brain MBP levels, the presence of labeled stem cells and their markers in several areas of the brain sections in hUCB- transplanted shiverer mice demonstrated that hUCB survived in vivo and migrated to various regions of brain within a week despite only being implanted at 1 site. The migrating capability of these hUCB is interesting in contrast to human glial progenitor cells, which were administered at 5 different sites of the shiverer CNS to achieve sufficiently widespread central myelination [26]. The results of the present study further strengthened our earlier reported findings on the in vivo survival and migration of hUCB in various animal models $[15,28]$.

The results of our present study have demonstrated for the first time that hUCB transplantation could be an effective means of treating hereditary hypomyelination and other demyelinating disorders. However, in this study we have not addressed the long-term survival of hUCB in the shiverer brains, the myelinating capability of transdifferentiated hUCB at various regions in shiverer CNS and overall survival time of the mice with neurological improvement. We continue to study the impact of hUCB 
implantation to neonatal shiverer mice on their survival and neurological recovery.

\section{Acknowledgments}

This research was supported by a grant from the Illinois Regenerative Medicine Institute, a program of the Illinois Department of Public Health funded through the Illinois Department of Healthcare and Family Services. We thank Peggy Mankin, Noorjehan Ali, and Robert Caughey for their technical assistance. We thank Shellee Abraham for article preparation and Diana Meister and Sushma Jasti for article review.

\section{Author Disclosure Statement}

No competing financial interests exist.

\section{References}

1. Horwitz EM, DJ Prockop, LA Fitzpatrick, WW Koo, PL Gordon, M Neel, M Sussman, P Orchard, JC Marx, RE Pyeritz and MK Brenner. (1999). Transplantability and therapeutic effects of bone marrow-derived mesenchymal cells in children with osteogenesis imperfecta. Nat Med 5:309-313.

2. Koc ON, SL Gerson, BW Cooper, SM Dyhouse, SE Haynesworth, AI Caplan and HM Lazarus. (2000). Rapid hematopoietic recovery after coinfusion of autologous-blood stem cells and culture-expanded marrow mesenchymal stem cells in advanced breast cancer patients receiving high-dose chemotherapy. I Clin Oncol 18:307-316.

3. Pittenger MF, AM Mackay, SC Beck, RK Jaiswal, R Douglas, JD Mosca, MA Moorman, DW Simonetti, S Craig and DR Marshak. (1999). Multilineage potential of adult human mesenchymal stem cells. Science 284:143-147.

4. Broxmeyer HE, GW Douglas, G Hangoc, S Cooper, J Bard, D English, M Arny, L Thomas and EA Boyse. (1989). Human umbilical cord blood as a potential source of transplantable hematopoietic stem/progenitor cells. Proc Natl Acad Sci U S A 86:3828-3832.

5. Rubinstein P, RE Rosenfield, JW Adamson and CE Stevens. (1993). Stored placental blood for unrelated bone marrow reconstitution. Blood 81:1679-1690.

6. Wexler SA, C Donaldson, P Ning-Kendall, C Rice, B Bradley and JM Hows. (2003). Adult bone marrow is a rich source of human mesenchymal stem cells but umbilical cord and mobilized adult blood are not. Br J Haematol 121: 368-374.

7. Moezzi L, AA Pourfathollah, K Alimoghaddam, M Soleimani and AR Ardjmand. (2005). The effect of cryopreservation on clonogenic capacity and in vitro expansion potential of umbilical cord blood progenitor cells. Transplant Proc 37: 4500-4503.

8. Kern S, H Eichler, J Stoeve, H Kluter and K Bieback. (2006). Comparative analysis of mesenchymal stem cells from bone marrow, umbilical cord blood, or adipose tissue. Stem Cells 24:1294-1301.

9. Garbuzova-Davis S, AE Willing, S Saporta, PC Bickford, C Gemma, N Chen, CD Sanberg, SK Klasko, CV Borlongan and PR Sanberg. (2006). Novel cell therapy approaches for brain repair. Prog Brain Res 157:207-222.

10. Friedman R, M Betancur, L Boissel, $H$ Tuncer, $C$ Cetrulo and H Klingemann. (2007). Umbilical cord mesenchymal stem cells: adjuvants for human cell transplantation. Biol Blood Marrow Transplant 13:1477-1486.

11. Yang SE, CW Ha, M Jung, HJ Jin, M Lee, H Song, S Choi, W Oh and YS Yang. (2004). Mesenchymal stem/progenitor cells developed in cultures from UC blood. Cytotherapy 6:476-486.

12. Saporta S, JJ Kim, AE Willing, ES Fu, CD Davis and PR Sanberg. (2003). Human umbilical cord blood stem cells infusion in spinal cord injury: engraftment and beneficial influence on behavior. J Hematother Stem Cell Res 12:271-278.

13. Kang KS, SW Kim, YH Oh, JW Yu, KY Kim, HK Park, CH Song and H Han. (2005). A 37-year-old spinal cord-injured female patient, transplanted of multipotent stem cells from human UC blood, with improved sensory perception and mobility, both functionally and morphologically: a case study. Cytotherapy 7:368-373.

14. Kuh SU, YE Cho, DH Yoon, KN Kim and Y Ha. (2005). Functional recovery after human umbilical cord blood cells transplantation with brain-derived neutrophic factor into the spinal cord injured rat. Acta Neurochir (Wien) 147:985-992.

15. Dasari VR, DG Spomar, CS Gondi, CA Sloffer, KL Saving, M Gujrati, JS Rao and DH Dinh. (2007). Axonal remyelination by cord blood stem cells after spinal cord injury. I Neurotrauma 24:391-410.

16. Dasari VR, DG Spomar, L Li, M Gujrati, JS Rao and DH Dinh. (2008). Umbilical cord blood stem cell mediated downregulation of Fas improves functional recovery of rats after spinal cord injury. Neurochem Res 33:134-149.

17. Peterson AC, GM Bray. (1984). Hypomyelination in the peripheral nervous system of shiverer mice and in shiverer in equilibrium normal chimaera. I Comp Neurol 227: 348-356.

18. Rosenbluth J. (1980). Peripheral myelin in the mouse mutant Shiverer. J Comp Neurol 193:729-739.

19. Bird TD, DF Farrell and SM Sumi. (1978). Brain lipid composition of the shiverer mouse: (genetic defect in myelin development). J Neurochem 31:387-391.

20. Rosenbluth J. (1980). Central myelin in the mouse mutant shiverer. J Comp Neurol 194:639-648.

21. Wrathall JR, W Li and LD Hudson. (1998). Myelin gene expression after experimental contusive spinal cord injury. J Neurosci 18:8780-8793.

22. Mitome M, HP Low, PA van den, JJ Nunnari, MK Wolf, S Billings-Gagliardi and WJ Schwartz. (2001). Towards the reconstruction of central nervous system white matter using neural precursor cells. Brain 124:2147-2161.

23. Nistor GI, MO Totoiu, N Haque, MK Carpenter and HS Keirstead. (2005). Human embryonic stem cells differentiate into oligodendrocytes in high purity and myelinate after spinal cord transplantation. Glia 49:385-396.

24. Yandava BD, LL Billinghurst and EY Snyder. (1999). “Global" cell replacement is feasible via neural stem cell transplantation: evidence from the dysmyelinated shiverer mouse brain. Proc Natl Acad Sci U S A 96:7029-7034.

25. Windrem MS, MC Nunes, WK Rashbaum, TH Schwartz, RA Goodman, G McKhann, NS Roy and SA Goldman. (2004). Fetal and adult human oligodendrocyte progenitor cell isolates myelinate the congenitally dysmyelinated brain. Nat Med 10:93-97.

26. Windrem MS, SJ Schanz, M Guo, GF Tian, V Washco, N Stanwood, M Rasband, NS Roy, M Nedergaard, LA Havton, S Wang and SA Goldman. (2008). Neonatal chimerization with human glial progenitor cells can both remyelinate and rescue the otherwise lethally hypomyelinated shiverer mouse. Cell Stem Cell 2:553-565. 
27. Veeravalli KK, VR Dasari, AJ Tsung, DH Dinh, M Gujrati, D Fassett and JS Rao. (2009). Stem cells downregulate the elevated levels of tissue plasminogen activator in rats after spinal cord injury. Neurochem Res 34:1183-1194.

28. Gondi CS, KK Veeravalli, B Gorantla, DH Dinh, D Fassett, JD Klopfenstein, M Gujrati and JS Rao. (2010). Human umbilical cord blood stem cells show PDGF-D-dependent glioma cell tropism in vitro and in vivo. Neuro Oncol 12:453-465.

29. Keilhoff G, F Stang, A Goihl, G Wolf and H Fansa. (2006). Transdifferentiated mesenchymal stem cells as alternative therapy in supporting nerve regeneration and myelination. Cell Mol Neurobiol 26:1235-1252.
Address correspondence to: Dr. Jasti S. Rao

Department of Cancer Biology and Pharmacology University of Illinois College of Medicine at Peoria

One Illini Drive

Peoria, IL 61656

E-mail: jsrao@uic.edu

Received for publication May 6, 2010

Accepted after revision October 6, 2010

Prepublished on Liebert Instant Online Month 00, 000 
